\title{
The Significance of Solutions Obtained from Ill-Posed Systems of Linear Equations Constituted by Synchrotron Radiation Based Anomalous Small-Angle X-Ray Scattering
}

\author{
Günter Johannes Goerigk \\ Institute of Soft Matter and Functional Materials, Helmholtz-Zentrum Berlin, Berlin, Germany \\ Email: guenter.goerigk@helm holtz-berlin.de
}

How to cite this paper: Goerigk, G.J. (2018) The Significance of Solutions Obtained from Ill-Posed Systems of Linear Equations Constituted by Synchrotron Radiation Based Anomalous Small-Angle X-Ray Scattering. Advances in Linear Algebra \& Matrix Theory, 8, 64-86.

https://doi.org/10.4236/alamt.2018.81007

Received: February 13, 2018

Accepted: March 24, 2018

Published: March 27, 2018

Copyright $\odot 2018$ by author and Scientific Research Publishing Inc. This work is licensed under the Creative Commons Attribution International License (CC BY 4.0). http://creativecommons.org/licenses/by/4.0/

\section{Open Access}

\begin{abstract}
Synchrotron radiation based experimental techniques known as Anomalous Small-Angle X-ray Scattering (ASAXS) provide deep insight into the nanostructure of uncountable material systems in condensed matter research i.e. solid state physics, chemistry, engineering and life sciences thereby rendering the origin of the macroscopic functionalization of the various materials via correlation to its structural architecture on a nanometer length scale. The techniques constitute a system of linear equations, which can be treated by matrix theory. The study aims to analyze the significance of the solutions of the stated matrix equations by use of the so-called condition numbers first introduced by A. Turing, J. von Neumann and H. Goldstine. Special attention was given for the comparison with direct methods i.e. the Gaussian elimination method. The mathematical roots of ill-posed ASAXS equations preventing matrix inversion have been identified. In the framework of the theory of von Neumann and Goldstine the inversion of certain matrices constituted by ASAXS gradually becomes impossible caused by non-definiteness. In Turing's theory which starts from more general prerequisites, the principal minors of the same matrices approach singularity thereby imposing large errors on inversion. In conclusion both theories recommend for extremely ill-posed ASAXS problems avoiding inversion and the use of direct methods for instance Gaussian elimination.
\end{abstract}

\section{Keywords}

Matrix Inversion, Condition Numbers, LU-Decomposition, Gaussian Elimination, Synchrotron Radiation, Anomalous Small-Angle X-Ray Scattering 


\section{Introduction}

In 1947 Alan Turing stated that the best known method for the solution of linear equations is Gauss's elimination method [1]. Turing reported that the elimination method "...unfortunately recently come into disrepute on the ground that rounding-off will give rise to very large errors" [1]. In the 1940s, the time just before electronic digital computers became available, a serious concern was felt, that rounding-off errors could drastically falsify the results obtained from such machines especially when dealing with complex mathematical algorithms like the inversion of a high-dimensional $n \times n$ matrix representing a system of linear equations (SLE). In this time $n=100$ was thought to be solvable with the new machines to come. In his paper Turing argued that from the practical work of L. Fox [2] in applying the elimination method no evidence for an exponential build-up of errors as suspected by Hotelling [3] was found. Moreover from his mathematical analysis Turing came to the result that “...in all normal cases the Hotelling estimate is far too pessimistic" [1]. Similar results have been outlined independently by J. von Neumann and H. Goldstine at the same time in their famous inversion paper [4]. Both studies represent a fundamental approach. Turing treated the problem from the most general point i.e. developing his theory for matrices with non-singular principal minors while von Neumann and Goldstine started from treating symmetric positive definite (SPD) matrices and subsequently extended their theory to non-definite matrices.

In this study we will make use of the concept of the condition numbers as introduced by Turing [1], von Neumann and Goldstine [4]. We will focus on $3 \times$ 3 matrices $(n=3)$ which are constituted by synchrotron radiation based condensed matter research using Anomalous Small-Angle X-ray Scattering though von Neumann and Goldstine stated explicitly, that the order of the matrix treated in their paper should be: $n \geq 10$. Their words [4]: "Indeed, for smaller values of $n$ the problem of inverting a matrix hardly justifies this thorough analysis". The reason why we apply the theories of von Neumann, Goldstine and Turing to matrices of low dimension $(n=3)$ is the extreme ill-conditioning of these low order matrices inherently introduced by scattering theory in synchrotron radiation, making it difficult to judge whether the obtained solutions respective matrix inversions carry significance or not. The theories of von Neumann, Goldstine and Turing shed light on the problem and tell how to define a figure of merit for results obtained from challenging synchrotron radiation experiments (ASAXS) in context with the mathematical algorithms being applied.

In the 70 years after Turing, von Neumann and Goldstine numerous publications established the scientific field of numeric mathematics dealing professionally with the problem of significance of the solutions obtained via matrix inversion, examples are [5] [6] [7] [8] [9]. But there are several reasons for referring preferentially to the two in the sense of numerical mathematics fundamental papers of Turing and von Neumann, Goldstine [1] [4]: 
1) Turing's definition of an ill-conditioned matrix (i.e. small percentage errors in the coefficients lead to large percentage errors in the solution) introduced in combination with the condition numbers the possibility to directly calculating the upper bound of the errors of the solution vector when stating rounding-off errors of a distinct size. This is done by the so-called backward error analysis first introduced in the publication of von Neumann and Goldstine [4]. In our paper we replace rounding-off errors by measurement errors and thus the synchrotron radiation based experiments can fully apply to the theories of Turing, von Neumann and Goldstine.

2) Turing came to the result that there is no reason to believe that direct methods have disadvantages when compared to linear-iterative methods for instance successive approximation. Following this idea, we will demonstrate that solving the linear problem via an analytic expression (if available) gives clear information about the significance by virtue of the backward error propagation, which is generally furnished throughout the formula of the used analytic expression.

3) Calculation of the inverse can produce large errors in the solution though the residual error is small. The fundamental publications of Turing and von Neumann, Goldstine gave at first a quantitative measure (figure of merit) for matrix inversion of ill-posed linear equations via condition numbers, but the condition numbers are different in the two publications due to their different approach. Until today these two concepts of quantification of conditioning (i.e. significance) represent reliable error estimations of ill-posed SLEs thereby providing a powerful tool to experimental physicists. Both concepts named in modern terminology $\mathrm{N}$-condition (name given by Turing) and $P$-condition (name not given by von Neumann, Goldstine) provide upper limits for the errors constituted by the SLEs and will be compared in this paper for ill-posed materials being subject to condensed matter research with synchrotron radiation.

Small-Angle X-ray Scattering (SAXS) experiments average over a large sample volume and give structural and quantitative information of high statistical significance on nanoscopic length scales between 1 and hundreds of nanometers because an enormous number of scattering entities up to $10^{12}$ is probed, which can be correlated to the macroscopic physical and chemical properties of the analyzed condensed matter systems in solid state physics, chemistry, engineering and life science. Detailed descriptions of the experimental and theoretical aspects of small-angle scattering can be found under [10] [11] [12]. By use of synchrotron radiation at suitable storage rings the so-called Anomalous Small-Angle X-ray Scattering (ASAXS) can be employed, which is an excellent tool for the chemical selective structural analysis of multi-component systems from various scientific fields like alloys, ceramics, magnetic systems, catalysts, semiconductors, glasses, polymers, technical membranes (for instance used in fuel cell applications) or bio-membranes (in pharmaceutical or cell research) and 
many other soft matter systems. Details about the ASAXS-techniques can be found in the reviews [13] [14]. In a former publication the solution of the eigenvector problem stated by the ASAXS techniques has been outlined in detail [15].

This publication aims to calculate the condition numbers as established by Turing and von Neumann, Goldstine for different solid state, catalytic and physicochemical systems from a selected series of ASAXS publications [16]-[30], which use direct methods (i.e. Gaussian elimination). The paper will compare the results of the Gaussian elimination with the predictions deduced from the condition numbers. Details about the scientific problems, which have been addressed by the ASAXS measurements, can be found in the related citations. We will not refer to ASAXS results from the decades before because in those days the separation of the pure-resonant scattering contribution was impossible (due to experimental limitations) meaning the vector equation was unsolvable due to the absence of the latter.

The paper explains why ASAXS studies potentially provide misleading results thereby at $1^{\text {st }}$ glance suggesting experimentally failing but at $2^{\text {nd }}$ glance indicting the application of inappropriate mathematical algorithms. This clear distinction became possible because the experimental accuracies of synchrotron radiation experiments (ASAXS) have been tremendously enhanced in the last decades thereby relocating the question of the significance of the deduced results to the mathematical algorithms. This study identifies the roots of possible mathematical mishandling of ASAXS problems owing to a lack of understanding concerning the nature of the involved SLEs thereby closing a gap in the literature clouding the ASAXS techniques.

\section{The General Matrix Equation Established by Anomalous Small-Angle X-Ray Scattering}

The remarkable possibilities of the ASAXS techniques are based on the energy dependence of the atomic scattering factors giving selective access to the specific SAXS contributions of the different chemical components in nano-scaled phases:

$$
f_{Z}(\varepsilon)=f_{0, Z}+f_{Z}^{\prime}(\varepsilon)+i f_{Z}^{\prime \prime}(\varepsilon)
$$

with $i$ being the imaginary unit. $Z$ represents the atomic number and $f_{Z}^{\prime}(\varepsilon), f_{Z}^{\prime \prime}(\varepsilon)$ are the anomalous dispersion corrections which depend generally on the energy $\varepsilon$. When performing ASAXS measurements on multi-component systems in the vicinity of the absorption edge of one of the sample constituents the scattering amplitude is generally:

$$
A(\overrightarrow{\mathbf{q}}, \varepsilon)=\int_{V_{p}} \Delta \rho_{A}(\overrightarrow{\mathbf{r}}, \varepsilon) \cdot \exp (i \overrightarrow{\mathbf{q}} \overrightarrow{\mathbf{r}}) \mathrm{d}^{3} r+\int_{V_{p}} \Delta \rho_{B}(\overrightarrow{\mathbf{r}}, \varepsilon) \cdot \exp (i \overrightarrow{\mathbf{q}} \overrightarrow{\mathbf{r}}) \mathrm{d}^{3} r
$$

where $q$ is the magnitude of the scattering vector $[(4 \pi / \lambda) \sin \Theta], 2 \Theta$ is the scattering angle, $\lambda$ the $\mathrm{X}$-ray wavelength and $V_{p}$ is the irradiated sample volume. $\Delta \rho_{A}, \Delta \rho_{B}$ are the differences of electron scattering length densities of the 
A-atoms and B-atoms (here A summarizes over all non-B atoms in the system),

$$
\begin{aligned}
& \Delta \rho_{A}(\overrightarrow{\mathbf{r}}, \varepsilon)=\Delta f_{A}(\varepsilon) \cdot v_{A}(\overrightarrow{\mathbf{r}})=\left(\left(f_{0, A}-\rho V_{A}\right)+f_{A}^{\prime}(\varepsilon)+i f_{A}^{\prime \prime}(\varepsilon)\right) \cdot v_{A}(\overrightarrow{\mathbf{r}}) \\
& \Delta \rho_{B}(\overrightarrow{\mathbf{r}}, \varepsilon)=\Delta f_{B}(\varepsilon) \cdot v_{B}(\overrightarrow{\mathbf{r}})=\left(\left(f_{0, B}-\rho V_{B}\right)+f_{B}^{\prime}(\varepsilon)+i f_{B}^{\prime \prime}(\varepsilon)\right) \cdot v_{B}(\overrightarrow{\mathbf{r}})
\end{aligned}
$$

calculated from the atomic (molecular) volumes $V_{A}, V_{B}$, respectively, where $\rho$ is the electron density of the entire sample. The functions $v_{A}(\overrightarrow{\mathbf{r}}), v_{B}(\overrightarrow{\mathbf{r}})$ are the number densities of the atomic (molecular) species $A$ and $B$, respectively and represent their spatial distribution in the sample. Calculating the scattering intensity $I(\overrightarrow{\mathbf{q}}, \varepsilon)=|A(\overrightarrow{\mathbf{q}}, \varepsilon)|^{2}=A(\overrightarrow{\mathbf{q}}, \varepsilon) \cdot A^{*}(\overrightarrow{\mathbf{q}}, \varepsilon)$ by means of Equations (2)-(3) and averaging over all orientations yields the sum of three scattering contributions,

$$
\begin{aligned}
& I(q, \varepsilon)=S_{A}(q, \varepsilon)+S_{A B}(q, \varepsilon)+S_{B}(q, \varepsilon) \quad[31]: \\
& S_{A}(q, \varepsilon)=\left|\Delta f_{A}(\varepsilon)\right|^{2} \cdot c_{A} \cdot\left|A_{A}(q)\right|^{2} \\
& S_{A B}(q, \varepsilon)=2\left[\left(f_{0, A}-\rho V_{A}+f_{A}^{\prime}(\varepsilon)\right)\left(f_{0, B}-\rho V_{B}+f_{B}^{\prime}(\varepsilon)\right)\right. \\
& \left.\quad+f_{A}^{\prime \prime}(\varepsilon) f_{B}^{\prime \prime}(\varepsilon)\right] \cdot c_{A B} \cdot \operatorname{Re}\left(A_{A}(q) A_{B}(q)\right) \\
& S_{B}(q, \varepsilon)=\left|\Delta f_{B}(\varepsilon)\right|^{2} \cdot c_{B} \cdot\left|A_{B}(q)\right|^{2} \\
& C_{A}=\varphi_{A}\left(1-\varphi_{A}\right) / V_{A}^{2}, c_{A B}=\varphi_{A} \varphi_{B} / V_{A} / V_{B}, c_{B}=\varphi_{B}\left(1-\varphi_{B}\right) / V_{B}^{2}
\end{aligned}
$$

$\varphi_{A}, \varphi_{B}$ are the volume fractions of the two components and thus related to the chemical concentrations. Because we want to analyze the conditioning of the ASAXS defined SLE in general, we have included the chemical concentrations into the pre-factors of Equation (4) that is in this paper the scattering functions of Equation (4) are based on the Fourier transform of the normalized pair correlation functions $\left|A_{A}(q)\right|^{2}, \operatorname{Re}\left(A_{A}(q) A_{B}(q)\right),\left|A_{B}(q)\right|^{2}$. The details are outlined in the Appendix 1.

The measurement of the scattering curves at different X-ray energies represented by index $i$ in the vicinity of the absorption edge of the B-atoms constitutes the following vector equation:

$$
\begin{aligned}
& \mathbf{M} \otimes \mathbf{A}=\mathbf{I} \\
& \mathbf{M}_{i j}\left(\varepsilon_{i}\right) \otimes \mathbf{A}_{j}(q)=\mathbf{I}_{i}\left(q, \varepsilon_{i}\right)
\end{aligned}
$$

where the summation is running over the index $j$ of the matrix (columns) and vector components. In order to simplify we will restrict the problem to the measurement of scattering curves at three X-ray energies: $i=1,2,3$. Measurements at more than 3 energies cannot improve the system of linear equations, because when measuring at $n$ energies in maximum 3 equations are linear independent while (n-3) equations are linear dependent [25]. The vector Equation (5) in explicit form writes:

$$
\left(\begin{array}{lll}
a_{11} & a_{12} & a_{13} \\
a_{21} & a_{22} & a_{23} \\
a_{31} & a_{32} & a_{33}
\end{array}\right) \otimes\left(\begin{array}{c}
\left|A_{A}(q)\right|^{2} \\
\operatorname{Re}\left(A_{A}(q) A_{B}(q)\right) \\
\left|A_{B}(q)\right|^{2}
\end{array}\right)=\left(\begin{array}{c}
I\left(q, \varepsilon_{1}\right) \\
I\left(q, \varepsilon_{2}\right) \\
I\left(q, \varepsilon_{3}\right)
\end{array}\right)
$$

with 


$$
\begin{aligned}
& a_{i 1}=\left[\left(\Delta f_{0, A}+f_{A}^{\prime}\left(\varepsilon_{i}\right)\right)^{2}+f_{A}^{\prime \prime}\left(\varepsilon_{i}\right)^{2}\right] c_{A} \\
& a_{i 2}=2\left[\left(\Delta f_{0, A}+f_{A}^{\prime}\left(\varepsilon_{i}\right)\right)\left(\Delta f_{0, B}+f_{B}^{\prime}\left(\varepsilon_{i}\right)\right)+f_{A}^{\prime \prime}\left(\varepsilon_{i}\right) f_{B}^{\prime \prime}\left(\varepsilon_{i}\right)\right] c_{A B} \\
& a_{i 3}=\left[\left(\Delta f_{0, B}+f_{B}^{\prime}\left(\varepsilon_{i}\right)\right)^{2}+f_{B}^{\prime \prime}\left(\varepsilon_{i}\right)^{2}\right] c_{B}
\end{aligned}
$$

and $\Delta f_{0, A, B}=f_{0, A, B}-\rho V_{A, B}$ which represent the electron density contrast of the components $\mathrm{A}$ and $\mathrm{B}$ with respect to the entire sample. Any non-singular real square matrix $\mathbf{M}$ may be decomposed into the product of two matrices:

$$
\mathbf{M}=\mathbf{L} \otimes \mathbf{U}
$$

where $\mathrm{L}$ and $\mathrm{U}$ mean an unique unit lower triangular and upper triangular matrix [1]:

$$
\begin{aligned}
& \left(\begin{array}{lll}
a_{11} & a_{12} & a_{13} \\
a_{21} & a_{22} & a_{23} \\
a_{31} & a_{32} & a_{33}
\end{array}\right)=\left(\begin{array}{ccc}
1 & 0 & 0 \\
l_{21} & 1 & 0 \\
l_{31} & l_{32} & 1
\end{array}\right) \otimes\left(\begin{array}{ccc}
u_{11} & u_{12} & u_{13} \\
0 & u_{22} & u_{23} \\
0 & 0 & u_{33}
\end{array}\right) \\
& l_{21}=\frac{a_{21}}{a_{11}}, l_{31}=\frac{a_{31}}{a_{11}}, l_{32}=\frac{a_{32}-\frac{a_{31}}{a_{11}} a_{12}}{a_{22}-\frac{a_{21}}{a_{11}} a_{12}}
\end{aligned}
$$

The unit lower matrix $\mathrm{L}$ represents the transformation of the vector Equations (6) by elementary operations (with $\operatorname{det}(\mathbf{L})=1$ ). The inverse matrix of $\mathbf{L}$ changes the matrix $\mathbf{M}$ into the upper triangular matrix $\mathrm{U}$ via:

$$
\mathbf{L}^{-1} \otimes \mathbf{M}=\mathbf{U}
$$

We introduce Equation (9b) into Equation (5) giving:

$$
\begin{aligned}
& \mathbf{L}^{-1} \otimes \mathbf{M} \otimes \mathbf{A}=\mathbf{L}^{-1} \otimes \mathbf{L} \otimes \mathbf{U} \otimes \mathbf{A}=\mathbf{U} \otimes \mathbf{A}=\mathbf{L}^{-1} \otimes \mathbf{I}=\mathbf{B} \\
& \mathbf{U}_{i j}\left(\varepsilon_{i}\right) \otimes \mathbf{A}_{j}(q)=\mathbf{B}_{i}\left(q, \varepsilon_{i}\right) \\
& \left(\begin{array}{ccc}
u_{11} & u_{12} & u_{13} \\
0 & u_{22} & u_{23} \\
0 & 0 & u_{33}
\end{array}\right)\left(\begin{array}{c}
\left|A_{A}(q)\right|^{2} \\
\operatorname{Re}\left(A_{A}(q) A_{B}(q)\right) \\
\left|A_{B}(q)\right|^{2}
\end{array}\right)=\left(\begin{array}{c}
B_{1} \\
B_{2} \\
B_{3}
\end{array}\right)
\end{aligned}
$$

where the meaning of $u_{i j}, B_{i}$ is explicitly outlined in the Appendix 1 (A3). The SLE in Equation (10) remains unchanged with respect to Equation (6) due to the elementary operations i.e. the solution vector is the same but we deal with a different vector $\mathbf{B}$ on the right side, which is a linear combination of the scattering curves in Equation (6).

The Equations (8)-(10) represent the most general mathematical description of any (non-contiguous) ASAXS problem in synchrotron radiation based experiments ${ }^{1}$. The diagonal elements of $U$ are (physical) invariants, more specifically the eigenvalues of the linear map, which are not changed by similarity transformations like the elementary operations. The matrix constituted by ASAXS is non-symmetric ${ }^{1}$ Equation (8) disregards permutations because partial pivoting turns out to be not necessary in the case of materials with non-contiguous X-ray absorption edges. Turing and von Neumann, Goldstine treated the most general case with permutations. 
and-more important-is in general not positive definite, due to possibly negative eigenvalues. We will come back to the latter when calculating the $P$-condition of von Neumann and Goldstine. In what is to follow we will focus on the vector Equation (10). As outlined in the Appendix 1 the restriction of the problem to $\mathrm{U}$ will not influence the findings of this publication.

\section{Systems being Capable of Using the Direct Method with Simplified Analytic Formula}

The upper triangular matrix $U$ is fully equivalent to the Gaussian elimination method and allows via backward substitution the analytic resolution of the SLE i.e. the Fourier transform of the pair correlation functions can be obtained via analytic formula as can be easily seen from the last line in vector Equation (10) giving:

$$
\left|A_{B}(q)\right|^{2}=B_{3} / u_{33}
$$

The quantity $B_{3}$ is directly related to the $3^{\text {rd }}$ component of the solution vector $\left|A_{B}(q)\right|^{2}$, which attracts the highest interest, because it carries exclusively all structural and quantitative (chemical concentration) information concerning the impact of the chemical component $B$ to the macroscopic functionalization of the material under investigation. Equation (11) combines scattering curves from different energies and allows the calculation of error propagation thereby giving the significance of the solution via error bars. Details about the mathematical algorithm employed in ASAXS techniques (Jacobian) are outlined in Appendix 2. As can be seen from the Equation (A3) the error propagation will behave in strongly different manner depending on the physical parameters say conditioning. It is calculated via the difference of the difference of two scattering curves weighted with pre-factors defined by the experiment, which amplify the error propagation. Typically the form factor of the pure-resonant scattering calculated via backward substitution is in the order of $10^{-3}$ to $10^{-2}$ with respect to the input scattering curves. This means the pre-factors define together with the measurement errors via amplification, whether a solution can carry significance or not. In other words, experiments which reduce the number of pre-factors or change their magnitude are better conditioned via reducing the amplification of error propagation thereby providing higher significance.

From Equation (A3) we learn, that via $u_{33}, B_{3}$ the 9 matrix coefficients $a_{i j}$ of Equation (7), which carry 2 contrast and 12 dispersion parameters (entering linearly and non-linearly the matrix coefficients) contribute 28 times as amplification factors into Equation (11) when calculating the form factor of the pure-resonant scattering. It is clear that this causes a large amplification of the error propagation when performing the backward substitution throughout a very complex formula.

We will now turn to experiments with better conditioning. A large number of two phase alloys for instance the semiconductor alloy silicon germanium have $\mathrm{X}$-ray absorption edges which lie far apart to each other. Moreover due to the 
light element silicon the anomalous dispersion corrections, $f_{S i}^{\prime}=f_{A}^{\prime} \approx 0$, $f_{S i}^{\prime \prime}=f_{A}^{\prime \prime} \approx 0$, can be taken as zero giving a tremendous simplification when inserting $u_{33}, B_{3}$ into Equation (11):

$$
\begin{aligned}
& \left|A_{B}(q)\right|^{2}=\left[\frac{I\left(q, \varepsilon_{1}\right)-I\left(q, \varepsilon_{2}\right)}{f_{B}^{\prime}\left(\varepsilon_{1}\right)-f_{B}^{\prime}\left(\varepsilon_{2}\right)}-\frac{I\left(q, \varepsilon_{1}\right)-I\left(q, \varepsilon_{3}\right)}{f_{B}^{\prime}\left(\varepsilon_{1}\right)-f_{B}^{\prime}\left(\varepsilon_{3}\right)}\right] \cdot \frac{1}{F\left(\varepsilon_{1}, \varepsilon_{2}, \varepsilon_{3}\right)} \\
& F\left(\varepsilon_{1}, \varepsilon_{2}, \varepsilon_{3}\right)=f_{B}^{\prime}\left(\varepsilon_{2}\right)-f_{B}^{\prime}\left(\varepsilon_{3}\right)+\frac{f_{B}^{\prime \prime 2}\left(\varepsilon_{1}\right)-f_{B}^{\prime \prime 2}\left(\varepsilon_{2}\right)}{f_{B}^{\prime}\left(\varepsilon_{1}\right)-f_{B}^{\prime}\left(\varepsilon_{2}\right)}-\frac{f_{B}^{\prime \prime 2}\left(\varepsilon_{1}\right)-f_{B}^{\prime \prime 2}\left(\varepsilon_{3}\right)}{f_{B}^{\prime}\left(\varepsilon_{1}\right)-f_{B}^{\prime}\left(\varepsilon_{3}\right)}
\end{aligned}
$$

Now only the dispersion parameters of component $B$ (here germanium) enter Equation (12) giving in total 6 dispersion parameters contributing 14 times in the amplification. Interestingly the contrast parameters vanished. In fact the conditioning is even better because in the energy range below the $\mathrm{K}$-absorption edge of germanium the $f_{G e}^{\prime \prime}\left(\varepsilon_{i}\right)=f_{B}^{\prime \prime}\left(\varepsilon_{i}\right) \approx 1 / 2$ are numbers of about $1 / 2$ giving very small numbers for the two decimal fractions in the second line of equation (12), which can be neglected. Thus only 3 dispersion parameters contribute substantially only 6 times. Moreover being able to neglect the decimal fractions the dispersion parameters enter all linearly thereby reducing the error propagation significantly. As a result such condensed matter systems can easily be analyzed via ASAXS experiments using the direct method of Equation (12) which represents Gaussian elimination [17] [22] [23] [25].

\section{Comparing the Results from the Direct Method with Expected Results from Matrix Inversion by Use of Condition Numbers}

In the previous section we have treated two-phase systems in solid state physics with ASAXS-constituted SLEs which have been successfully analyzed by Gaussian elimination. In concern of the direct method the situation is similar for a $2^{\text {nd }}$ material class. These materials are highly diluted three phase systems from soft matter research for instance polymer ions decorated by counter ions. The counter ions represent the phase $\mathrm{B}$ (for instance $\mathrm{Sr}^{2+}, \mathrm{Pb}^{2+}, \mathrm{Tl}^{+}, \mathrm{Br}^{-}, \mathrm{I}^{-}$ions), while the other two phases are the solvent (water or other fluids with low molecular weight) and the soft matter component (for instance polyacrylate, proteins or liposomes). Because the solvent and soft matter components consist of light elements the same arguments as for the two phase alloys with light elements hold and the simplified formula in Equation (12) can be applied with the exception that in the case of the $\mathrm{Pb}^{2+}$ and $\mathrm{Tl}^{+}$counter ions the decimal fractions no longer can be neglected because the $f_{P b}^{\prime \prime}\left(\varepsilon_{i}\right)=f_{B}^{\prime \prime}\left(\varepsilon_{i}\right)$ cover values between 4 and 6 when performing ASAXS measurements in the energy range of the $\mathrm{L}_{\mathrm{III}}$-absorption edge [16] [18] [19] [20] [24] [26] [27] [28] [30]. Other three phase systems come from catalyst research and can also be treated via Equation (12) for instance porous substrate structures (silica, carbon, or gibbsite and bayerite) for metal or metal oxide catalysts. The $3^{\text {rd }}$ phase is represented by vacuum (pores) [21] [29]. 
Though the direct method was successfully applied for all these materials it appeared that these systems behaved cumbersome in the ASAXS experiments, which was evidenced by numerous experimental parameters. For instance the primary flux normalization had to be more accurate, the transmission corrections needed to be improved to the accuracy regime of $10^{-5}$, additionally the absolute calibration of the scattering curves needed to be improved significantly, the beam stability needed substantial improvement etc. [14]. The reason of these systems being extra demanding may be attributed to various material parameters for instance high dilutions or small or vanishing contrast in the soft matter systems or a large contrast in combination with a high volume fraction of the pores (causing overwhelming pore scattering) or a small amount of the metal or ceramic component in the case of the catalyst systems.

A $3^{\text {rd }}$ material class is represented by three-phase systems with contiguous lying X-ray absorption edges. An example for such very ill-conditioned systems is $\mathrm{IrO}_{2}$ catalyst prepared on the semiconductor substrate $\mathrm{TaON}$ for solar fuel applications [32]. This material is hard to treat with ASAXS experiments because 1) the $\mathrm{L}_{\mathrm{II}}$-absorption edge of tantalum and the $\mathrm{L}_{\mathrm{III}}$-absorption of iridium lie close together i.e. Equation (12) cannot be used, 2) the substrate carries an enormous contrast with respect to vacuum (pore structure) due to the heavy element tantalum thereby causing an overwhelming pore scattering showing strong energy dependence in the energy range of the $\mathrm{L}_{\mathrm{III}}$-absorption edge of iridium. In summary this gives a worst case behavior making total pivoting potentially necessary, which we will not treat in this publication.

These very different examples illustrate, that we ought to know more about the conditioning of an ASAXS experiment so that we could better optimize the experimental parameters like the length of exposure times which govern the error of the scattering curves, the selection of X-ray energies, the accuracy required for the transmission measurements, the number of calibration measurements, the detector to be used and many other questions which appear, in advance of a beam time at $3^{\text {rd }}$ generation synchrotron radiation sources.

What we would like to have is say a figure of merit, which summarizes all these questions in one or two numbers and consequently define how to perform the experiment. This request is met by the condition numbers of Turing and von Neumann, Goldstine as will be shown in what is to follow.

Turing introduced the $\mathrm{N}$-condition number (in literature also known as Turing number) based on the Frobenius norm $\|\mathbf{U}\|$ :

$$
\begin{aligned}
& N(\mathbf{U})=\frac{1}{3} \cdot\|\mathbf{U}\| \cdot\left\|\mathbf{U}^{-1}\right\| \\
& \|\mathbf{U}\|=\sqrt{\sum_{i, j=1}^{3} u_{i j}^{2}},\left\|\mathbf{U}^{-1}\right\|=\sqrt{\sum_{i, j=1}^{3} \mu_{i j}^{2}}
\end{aligned}
$$

The $\mu_{i j}$ represent the coefficients of the inverse matrix. From this the backward error $\|\Delta A\| /\|A\|$ of the solution vector can be calculated via the error of the scattering curves $\|\Delta B\| /\|B\|[1]$ 


$$
\frac{\|\Delta A\|}{\|A\|} \leq 3 \cdot N(\mathbf{U}) \cdot \frac{\|\Delta B\|}{\|B\|}
$$

where

$$
\|\Delta B\|=\sqrt{\sum_{i=1}^{3} \Delta B_{i}^{2}},\|B\|=\sqrt{\sum_{i=1}^{3} B_{i}^{2}}
$$

and

$$
\|\Delta A\|=\sqrt{\sum_{i=1}^{3} \Delta A_{i}^{2}}, \quad\|A\|=\sqrt{\sum_{i=1}^{3} A_{i}^{2}}
$$

As an example we assume to have measured scattering curves with a relative accuracy of $\|\Delta B\| /\|B\|=10^{-3}$ via ASAXS on semiconductor alloys with a $N$-condition number $N(\mathbf{U})=30$. Inserting these numbers into Equation (14) gives $\|\Delta A\| /\|A\|=0.09$, meaning that the summarized error of the components of the solution vector obtained by matrix inversion achieve relative accuracies within $9 \%$. This result tells that the solution vector deduced from inversion carries significance, which we have been expecting because our backward error propagation calculation via the simplified Equation (12) gave already a better result due to establishing for each single vector component the criterion of significance. The latter cannot be rendered by the $\mathrm{N}$-condition number, because it deals with the ratio of the square root of summed squares thereby giving only an upper limit for the error.

But the condition number provides at this point the invaluable information that the error estimations from matrix theory and error propagation are similar for these materials thereby suggesting that this material class can be treated by ASAXS with a high degree of significance in both directions via back substitution (Gaussian elimination) or alternatively matrix inversion. In other words Turing's theory states that these systems behave well because the analytic calculations of error propagation and error estimation via condition numbers are close together. The details come from the calculation of error propagation while the universal information comes from the linear theory but for these materials they nearly coincide! Analysis and linear algebra go together.

\section{Condition Numbers of Two-Phase Systems}

Figure 1 depicts $N(\mathrm{U})$ for materials with $Z$ between sodium and uranium on the $x$-axis embedded in host materials with electron densities between $1.0 \times 10^{23}(\approx$ lithium) and $2.6 \times 10^{24}$ ( $\approx$ molybdenum) electrons $/ \mathrm{cm}^{3}$ on the $y$-axis. Details of the calculations are outlined in Appendix 3. The color code represents the logarithm naturalis of the $N$-condition number. Figure 1 (a) depicts the $N$-condition number for atomic concentrations of $50 \%$ i.e. $x=0.5$ showing an increase from low to high $Z$. The red line crossing in the middle of the picture is the $10 \%$-line (corresponding to $\mathrm{N}$-condition number of 34 ), where due to Equation (14) the error of the solution vector is $10 \%$ when assuming scattering 


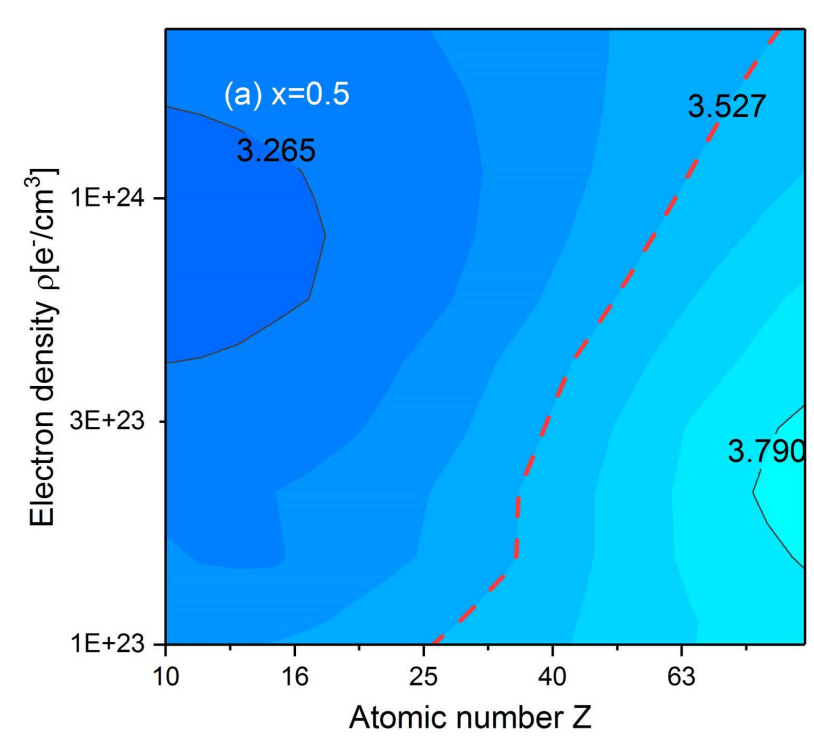

$\ln \mathrm{N}$
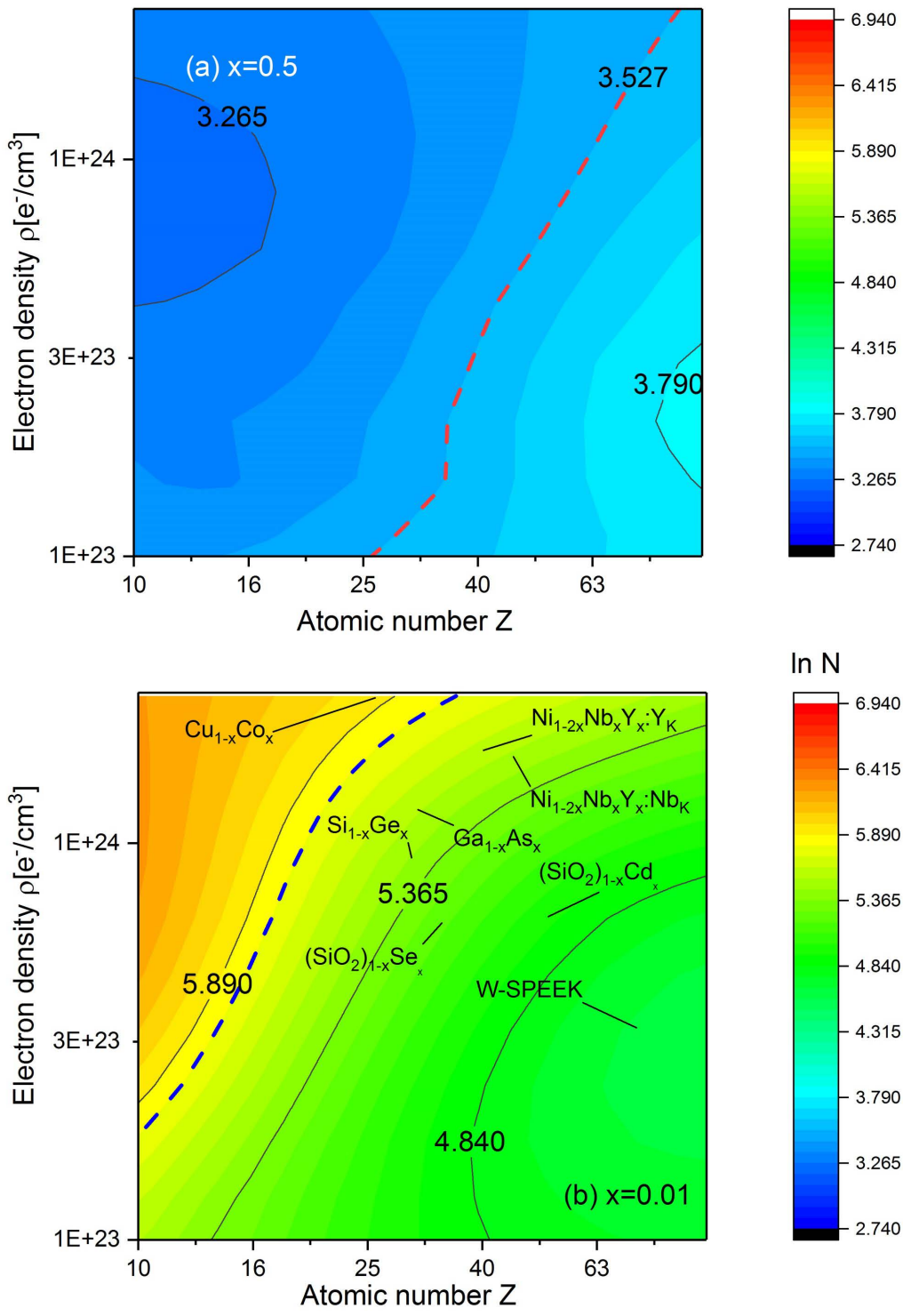

In $\mathrm{N}$

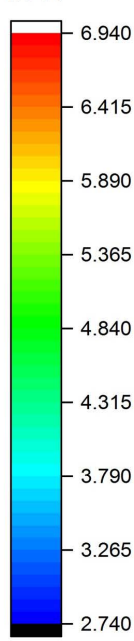

Figure 1. The $\mathrm{N}$-condition number of two-phase systems for (relative) number densities of $50 \%$ (a) and $1 \%$ (b). The color code represents the natural logarithm of the $N$-condition. The red dashed line in (a) represents $N=34$ i.e. the relative error of the solution vector amounts $10 \%$. The blue dashed line in (b) represents $N=331$ giving the border of insignificance (see text). In (b) several examples from the literature are depicted.

curves with $\|\Delta B\| /\|B\|=10^{-3}$. Thus at the concentration $x=0.5$ for nearly all alloys the solution vector obtained from matrix inversion will provide high significance. The latter represents a comment on why alloys became the first systems quantitatively analyzed by ASAXS in the 1990s.

The situation appears different for small concentrations $(x=0.01)$ as depicted in Figure 1(b). From right bottom to top left the $N$-condition number increases. The blue line crossing is the border of resolution corresponding to $N$-condition number of 331 meaning that the relative error $\|\Delta A\| /\|A\|$ approaches 1 
(insignificance) when having $\|\Delta B\| /\|B\|=10^{-3}$. To gain the solution vector now via matrix inversion will mean to improve drastically the accuracy of measured scattering curves into the $10^{-4}$ regime i.e. extending the exposure (measurement) time by a factor of 100 ! The $N$-condition approaches very large values (upper left of Figure 1(b)) because the principal minors of the matrix approach zero due to the small volume fractions (chemical concentrations) $\varphi_{A}, \varphi_{B}$.

Several examples from the literature are explicitly depicted in Figure 1(b): crystalline copper-cobalt alloys, amorphous silicon germanium semiconductor alloys (photovoltaics) [17], $\mathrm{CdS}_{\mathrm{x}} \mathrm{Se}_{1-\mathrm{x}}$ quantum dots in a silica glass matrix (optical filters), ternary alloys exhibiting spinodal decomposition [22] and tungsten embedded in proton conducting SPEEK (sulfonated-polyether-ether-ketone) membranes used in fuel cell techniques. Figure 2 summarizes for the two-phase systems $N$-condition maps for different (relative) atomic concentrations $X=0.01$, $0.27,0.5,0.7$.

\section{Condition Numbers of Three-Phase Systems}

Figure 3(a) depicts $N(\mathrm{U})$ for three-phase systems from soft matter research. The $x$-axis shows like in the previous section the atomic number $Z$. On the $y$-axis the absolute value of the electron contrast between the molecular system and the solvent is plotted in units of electrons. The absolute value is needed because the plot is logarithmic thus including negative and positive contrasts. Three examples are shown in the map. These are negatively charged polyacrylate chains highly diluted in water decorated with the two different counter ions $\mathrm{Sr}^{2+}$ and $\mathrm{Pb}^{2+}$ with a concentration of $1.5 \mathrm{mM}$ [20] [30]. For the contrast we assume two values 1 and 0.1 electron. As can be seen for the contrast 1 the $N$-condition

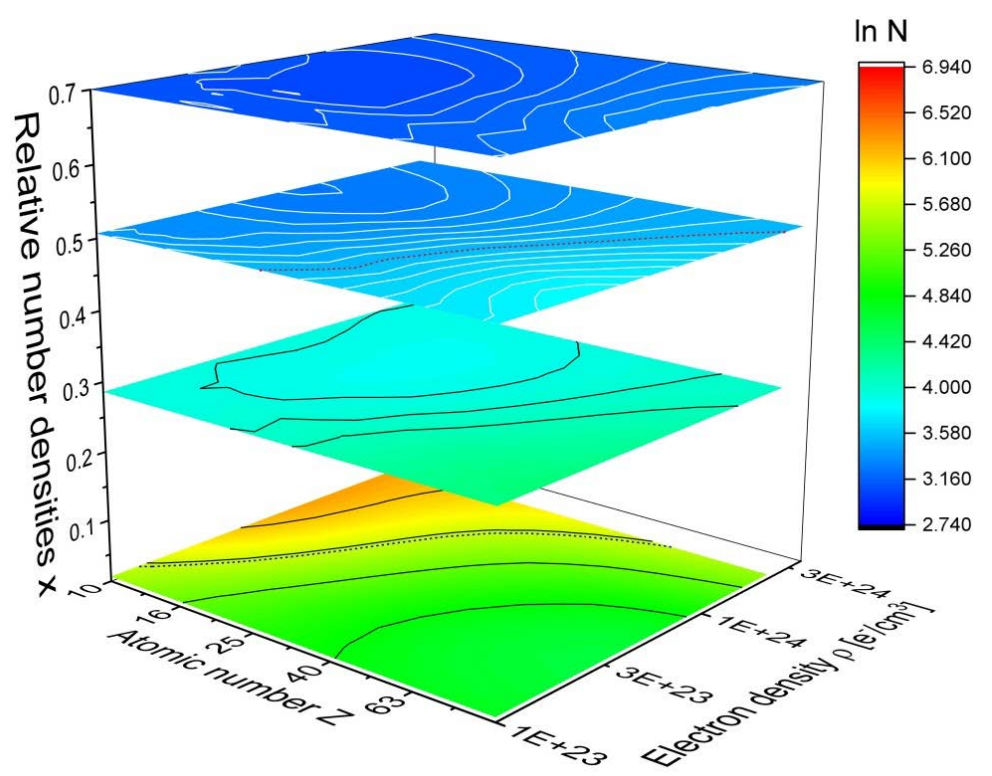

Figure 2. The $N$-condition number of two-phase systems for (relative) number densities of $70 \%, 50 \%, 27 \%$ and $1 \%$. The maps for $50 \%$ and $1 \%$ are the same as in Figure 1. 


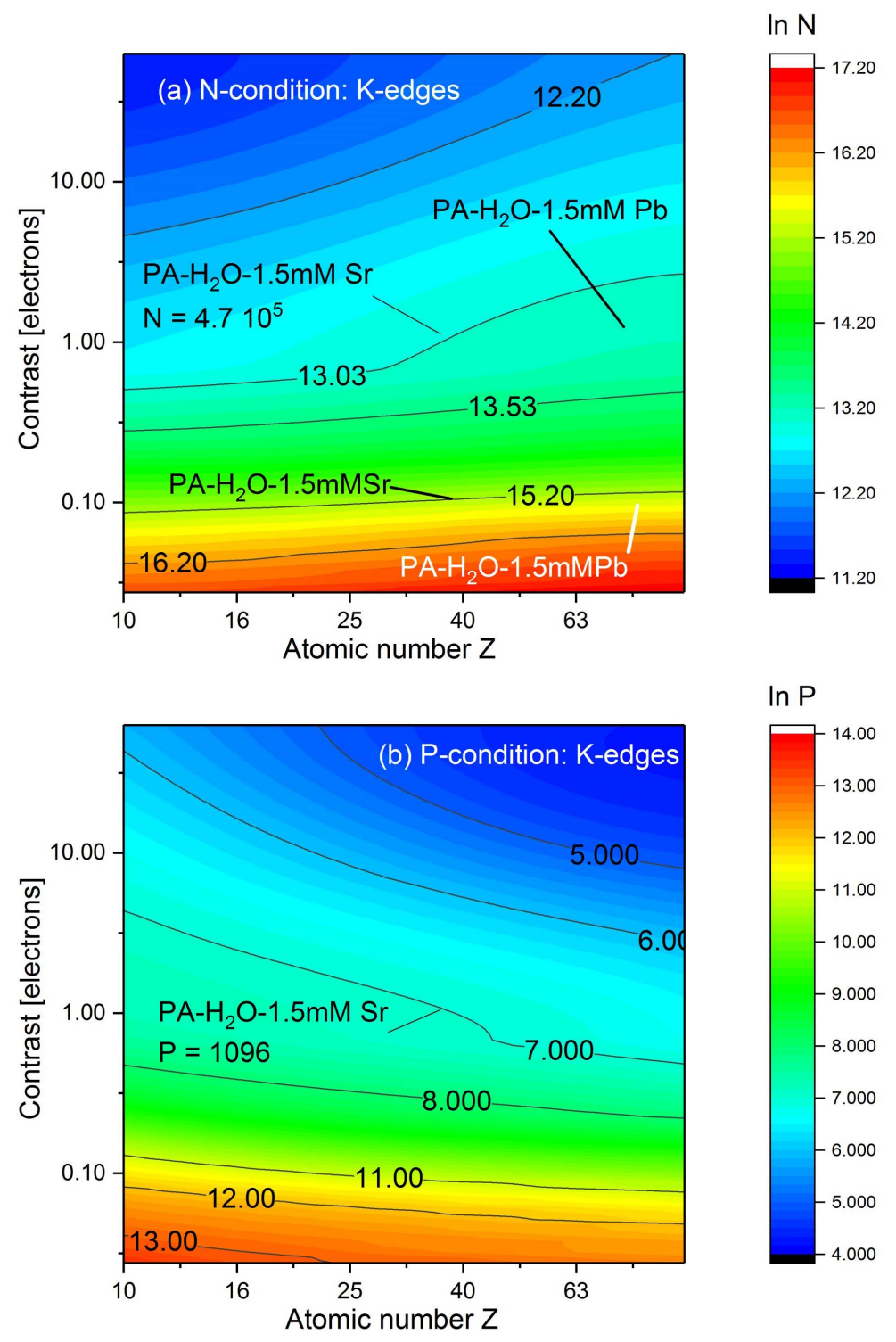

Figure 3. The $N$-condition number (a) of diluted three-phase systems. On the $y$-axis the contrast is plotted logarithmically i.e. includes also negative contrast. The units are electrons. The examples are from the literature [20] [26] [30] showing $\mathrm{Sr}^{2+}$ and $\mathrm{Pb}^{2+}$ counter ions with a concentration of $1.5 \mathrm{mM}$ decorating polyacrylate anions in $\mathrm{H}_{2} \mathrm{O}$. The $P$-condition number of the same systems is plotted in (b). For small contrast the condition numbers become extremely large indicating that matrix inversion lost significance (see text). For the $\mathrm{Pb}$ containing system the markers in the map give only a rough estimation of the condition numbers because the maps need to be re-calculated for $\mathrm{L}_{\mathrm{III}}$-absorption edges (see Appendix 3).

number of the $\mathrm{Sr}$ containing system reaches a value of about $4.7 \times 10^{5}$ i.e. the insignificance amounts to $\|\Delta A\| /\|A\|=1400$ !! For a contrast of 0.1 electrons $N$ becomes even larger. According to Equation (14) apparently we have crossed the border of insignificance. The reasons are the very low chemical concentrations of the counter ions thereby causing some of the principal minors approaching 
singularity. The outcome from the large $N$-condition number is that a matrix inversion in such cases never can provide a significant solution vector. This seems to be in contradiction to results from the literature where significant results have been reported via employment of Gaussian elimination using Equation (12) [16] [20] [26] [27] [28] [30]. The results have been verified carefully via error propagation calculation suggesting a mysterious contradiction between $\mathrm{N}$-condition number used in linear algebra and error calculation in analysis. Of course the $\mathrm{N}$-condition is an upper limit in the sense of the largest possible error. But the large discrepancy leads into contemplation.

In order to obtain additional information we now introduce the $P$-condition number of von Neumann and Goldstine:

$$
P(\mathbf{U})=\frac{\left|\lambda_{\max }\right|}{\left|\lambda_{\min }\right|}
$$

The $\left|\lambda_{\max }\right|,\left|\lambda_{\min }\right|$ mean the eigenvalues of the matrix $U$ with the largest respective smallest absolute value. Figure 3 (b) depicts the $P$-condition for the soft matter three-phase systems. We receive for polyacrylate in water with 1.5 $\mathrm{mM} \mathrm{Sr}{ }^{2+}$ counter ions at a contrast of 1 electron $P=1096$. von Neumann and Goldstine introduced for the $P$-condition the following error estimation for solving the problem by matrix inversion [4]:

$$
\frac{\|\Delta A\|}{\|A\|}=14.24 P(\mathbf{U}) n^{2} \frac{\|\Delta B\|}{\|B\|}
$$

Because the order of our matrix is $n=3$ we receive via substitution the relative error for the solution vector of $\|\Delta A\| /\|A\|=140$ when assuming $\|\Delta B\| /\|B\|=10^{-3}$. Thus von Neumann and Goldstine find insignificance as well but not so drastic as Turing. The explanation for the discrepancy comes from the fact that the matrices constituted by ASAXS are not necessarily positive definite. Turing's $\mathrm{N}$-condition number tolerates negative eigenvalues (see Figure 1(a) depicting in the upper left systems with negative contrast) because his theory is based on most general (non-singular) matrices but it approaches large values when the principal minors approach zero (see Figure 1(b) which in the upper left represents systems with small chemical concentrations $x=0.01$ ). In case of the three-phase soft matter systems there are two reasons why the $N$-condition drastically increases. One is the small chemical concentration the other a vanishing electron contrast between the soft matter component and the solvent i.e. small numbers of electrons on the $y$-axis in Figure 3(a). Both parameters are multiplied when entering the matrix coefficients in Equation (7). The situation is different for von Neumann's and Goldstine's $P$-condition.

Equation (18) was introduced by von Neumann and Goldstine explicitly for positive definite matrices. For non-definite matrices their a priori error estimation of Equation (18) turned into the a posteriori error estimation [4]:

$$
\frac{\|\Delta A\|}{\|A\|}=36.58 P(\mathbf{U})^{2} n^{2} \frac{\|\Delta B\|}{\|B\|}
$$


Note the $P$-condition now enters squared into Equation (19). This gives $\|\Delta A\| /\|A\|=395464$ i.e. a value of insignificance now drastically larger than Turing's estimation based on $\mathrm{N}$-condition. In consequence the theories of von Neumann, Goldstine and Turing accord in stating that in the case of such (soft matter) low dimensional $(n=3)$ matrices constituted by ASAXS matrix inversion is not capable to provide any reliable solution vectors when dealing with measurement errors of $\|\Delta B\| /\|B\|=10^{-3}$. The calculation of the inverse produces large errors in the solution though the residual error is small. The only way to escape from this problem is drastically enhancing the significance of the input data (i.e. reducing the error of the measured scattering curves) to a degree dictated by the condition numbers, which of course faces limitations due to the experimental techniques. In order to reach the border of significance for inversion (based on Turing's theory) an accuracy of $\|\Delta B\| /\|B\|=10^{-6}$ is here required (exposure time $10^{6}$ times longer!!) which up to now by no means can be furnished by $3^{\text {rd }}$ generation synchrotron radiation sources.

\section{Discussion and Future Prospects}

von Neumann and Goldstine have been able to explain in detail-using an argumentation of reversed logic-that the absence of positive definiteness for matrices means that these matrices loose the non-singularity i.e. the calculation of the inverse becomes gradually impossible. In other words in case of the ill-posed soft matter systems we approach in the space of matrices the border line between non-singularity and singularity i.e. between matrices having an inverse or not. This corresponds in Turing's theory to the principal minors approaching singularity thereby violating the pre-requisite for the LU decomposition. This is what can be identified as the mathematical roots of the ASAXS problem of ill-posed systems!

The by far most interesting result of this study is that the direct method of Gaussian elimination succeeds via back substitution even in the case of such ill-posed soft matter systems where inversion fails. The Figures 1-3 can serve in future condensed matter research using synchrotron radiation based anomalous scattering techniques for the estimation of $N$-condition of the related scientific problem thereby gaining information whether matrix inversion can be used appropriately or seek for an analytic expression should be favored.

A further result is a clear warning to introduce iterative non-linear procedures (for instance via minimizing the residual error) into the data analysis of ASAXS measurements because these are by no means capable in giving any information about the significance of the solution. More specifically the information of significance is lost when employing non-linear algorithms because the linear theories of von Neumann, Goldstine and Turing are not applicable and thus per definitionem no criteria exist to judge significance i.e. these methods can be to a high degree misleading.

We want to outline the latter using a vector example taken from the text book 
of Joan Westlake [33]. Consider the following SLE in the two-dimensional space:

$$
\begin{aligned}
& x_{1}+10 x_{2}=11 \\
& 10 x_{1}+101 x_{2}=111
\end{aligned}
$$

or in terms of vector equations:

$$
\left(\begin{array}{cc}
1 & 10 \\
10 & 101
\end{array}\right) \otimes\left(\begin{array}{l}
x_{1} \\
x_{2}
\end{array}\right)=\left(\begin{array}{c}
11 \\
111
\end{array}\right)
$$

The solution vector is $x_{1}=1, x_{2}=1$. We now consider an approximate solution vector $x_{1}=1.001, x_{2}=1.01$. From Equation (21) the residual vector $\mathbf{r}$ with the components $r_{1}=0.101, r_{2}=1.02$ can be calculated giving a square root of the sum of squared residuals of

$$
\Delta r=\sqrt{\sum_{i=1}^{2} r_{i}^{2}}=1.025
$$

Now we consider a $2^{\text {nd }}$ vector with components $x_{1}=11.1, x_{2}=0$ giving the residual vector components $r_{1}=0.1, r_{2}=0$ with the square root of the sum of squared residuals $\Delta r=0.1$. The residual of the second vector is drastically smaller when compared to the residual of the first vector but the first vector is clearly closer to the true solution. The example demonstrates that non-linear fitting of a linear problem can fail drastically. The words of Joan Westlake: "Hence a residual of zero implies the solution is exact, but a residual vector that is small does not necessarily imply the solution is close. A small residual means there are no substantial errors in the direction of the large eigenvectors, but there may be very substantial errors in the direction of the small eigenvectors" [33]. In our case this means very substantial errors in the direction of the small eigenvector of the pure-resonant scattering in comparison to non-substantial errors of the large eigenvector of non-resonant scattering or vice versa. Of course the situation is more complicated because we have a $3^{\text {rd }}$ eigenvector represented by the mixed-resonant scattering.

\section{Conclusion}

The problem of ill-posed systems of linear equations constituted by Anomalous Small-Angle X-ray Scattering in synchrotron radiation is inherently introduced by scattering theory thereby prohibiting matrix inversion for numerous systems in condensed matter research. The mathematical roots for extreme ill-posed systems have been identified in the absence of positive definiteness of their matrices (von Neumann, Goldstine) or correspondingly the principal minors approaching singularity (Turing). Gaussian elimination can overcome this problem for numerous (not all) systems represented by a simplified analytic expression. This is evidenced by error propagation analysis thereby establishing significance. Only linear algorithms can give a figure of merit for the significance of the solutions. Minimizing the residuals can be to a high degree misleading. The study evidences that the significance of the deduced conclusions from ASAXS measurements depends not only on the accuracy of the experimental 
data but inevitably demands for the application of appropriate (linear) mathematical algorithms. High accuracies require appropriate algorithms.

\section{References}

[1] Turing, A.M. (1948) Rounding-off Errors in Matrix Processes. The Quarterly Journal of Mechanics and Applied Mathematics, 1, 287-308. https://doi.org/10.1093/qjmam/1.1.287

[2] Fox, L., Huskey, H.D. and Wilkinson, J.H. (1948) Notes on the Solution of Algebraic Linear Simultaneous Equations. The Quarterly Journal of Mechanics and Applied Mathematics, 1, 149-173. https://doi.org/10.1093/qjmam/1.1.149

[3] Hotelling, H. (1943) Some New Methods in Matrix Calculation. The Annals of Mathematical Statistics, 14, 1-34. https://doi.org/10.1214/aoms/1177731489

[4] von Neumann, J. and Goldstine H.H. (1947) Numerical Inverting of Matrices of High Order. Bulletin of the American Mathematical Society, 53, 1021-1100. https://doi.org/10.1090/S0002-9904-1947-08909-6

[5] Todd, J. (1949) The Condition of Certain Matrices I. The Quarterly Journal of Mechanics and Applied Mathematics, 2, 469-472.

https://doi.org/10.1093/qjmam/2.4.469

[6] Newman, M. and Todd, J. (1958) The Evaluation of Matrix Inversion Programs. Journal of the Society for Industrial and Applied Mathematics, 6, 466-476. https://doi.org/10.1137/0106030

[7] Wilkinson, J.H. (1972) Note on Matrices with a Very Ill-Conditioned Eigenproblem. Numerische Mathematik, 19, 176-178. https://doi.org/10.1007/BF01402528

[8] Symm, H.J. and Wilkinson, J.H. (1980) Realistic Error Bounds for a Simple Eigenvalue and Its Associated Eigenvector. Numerische Mathematik, 35, 113-126. https://doi.org/10.1007/BF01396310

[9] Olver, J.H. and Wilkinson, F.W. (1982) A Posteriori Error Bounds for Gaussian Elimination. Journal of Numerical Analysis, 2, 377-406.

[10] Guinier, A. and Fournet, G. (1955) Small-Angle Scattering of X-Rays. Wiley-VCH Verlag GmbH \& Co. KGaA, New York.

[11] Glatter, O. and Kratky, O. (1982) Small-Angle X-Ray Scattering. Academic Press, London.

[12] Kratky, O. (1983) The World of Negligible Dimensions and Small Angle Diffraction of X-Rays and Neutrons on Biological Macromolecules. Acta Leopoldina, 55, 6-72.

[13] Goerigk, G., Haubold, H.-G., Lyon, O. and J.-P. Simon J.-P. (2003) Anomalous Small-Angle X-Ray Scattering in Materials Science. Journal of Applied Crystallography, 36, 425-429. https://doi.org/10.1107/S0021889803000542

[14] Goerigk, G., Huber, K., Mattern, N. and Williamson, D.L. (2012) Quantitative Anomalous Small-Angle X-Ray Scattering-The Determination of Chemical Concentrations in Nano-scaled Phases. The European Physical Journal Special Topics, 208, 259-274. https://doi.org/10.1140/epjst/e2012-01623-2

[15] Goerigk, G.J. (2013) The Solution of the Eigenvector Problem in Synchrotron Radiation Based Anomalous Small-Angle X-Ray Scattering. Advances in Linear Algebra \& Matrix Theory, 3, 59-68. https://doi.org/10.4236/alamt.2013.34012

[16] Goerigk, G., Schweins, R., Huber, K. and Ballauff M. (2004) The Distribution of Sr ${ }^{2+}$ Counterions around Polyacrylate Chains Analyzed by Anomalous Small-Angle X-Ray Scattering. Europhysics Letters, 66, 331-337. https://doi.org/10.1209/epl/i2003-10215-y 
[17] Goerigk, G. and Williamson, D.L. (2006) Temperature Induced Differences in the Nanostructure of Hot-Wire Deposited Silicon-Germanium Alloys Analyzed by Anomalous Small-Angle X-Ray Scattering. Journal of Applied Physics, 99, 1-13. https://doi.org/10.1063/1.2187088

[18] Bóta, A., Varga, Z. and Goerigk, G. (2007) Biological Systems as Nanoreactors: Anomalous Small-Angle Scattering Study of the CdS Nanoparticle Formation in Multilamellar Vesicles. The Journal of Physical Chemistry B, 111, 1911-1915. https://doi.org/10.1021/jp067772n

[19] Varga, Z., Bóta, A. and Goerigk G. (2007) Localization of Dihalogenated Phenols in Vesicle Systems Determined by Contrast Variation X-Ray Scattering. Journal of Applied Crystallography, 40, 205-208. https://doi.org/10.1107/S0021889807001987

[20] Goerigk, G., Huber, K. and Schweins R. (2007) Probing the Extent of the $\mathrm{Sr}^{2+}$ Ion Condensation to Anionic Polyacrylate Coils: A Quantitative Anomalous Small-Angle X-Ray Scattering Study. The Journal of Chemical Physics, 127, 154908-1. https://doi.org/10.1063/1.2787008

[21] Bóta, A., Varga, Z. and Goerigk, G. (2008) Structural Description of the Nickel Part of a Raney-type Catalyst by Using Anomalous Small-Angle X-Ray Scattering. The Journal of Physical Chemistry C, 112, 4427-4429. https://doi.org/10.1021/jp800237b

[22] Goerigk, G. and Mattern, N. (2009) Critical Scattering of Ni-Nb-Y Metallic Glasses Probed by Quantitative Anomalous Small-Angle X-Ray Scattering. Acta Materialia, 57, 3652-3661. https://doi.org/10.1016/j.actamat.2009.04.028

[23] Goerigk, G. and Mattern, N. (2010) Spinodal Decomposition in Ni-Nb-Y Metallic Glasses Analyzed by Quantitative Anomalous Small-Angle X-Ray Scattering. Journal of Physics: Conference Series, 247, 012022. https://doi.org/10.1088/1742-6596/247/1/012022

[24] Akiba, I., Takechi, A., Sakou, M., Handa, M., Shinohara, Y., Amemiya, Y., Yagi, N. and Sakurai, K. (2012) Anomalous Small-Angle X-Ray Scattering Study of Structure of Polymer Micelles Having Bromines in Hydrophobic Core. Macromolecules, 45, 6150-6157. https://doi.org/10.1021/ma300461d

[25] Goerigk, G.J. (2013) The Impact of the Turing Number on Quantitative ASAXS Measurements of Ternary Alloys. JOM, 65, 44-53. https://doi.org/10.1007/s11837-012-0451-9

[26] Lages, S., Goerigk, G. and Huber, K. (2013) SAXS and ASAXS on Dilute Sodium Polyacrylate Chains Decorated with Lead Ions. Macromolecules, 46, 3570-3580. https://doi.org/10.1021/ma400427d

[27] Sakou, M., Takechi, A., Murakami, S., Sakurai, K. and Akiba, I. (2013) Study of the Internal Structure of Polymer Micelles by Anomalous Small-Angle X-Ray Scattering at Two Edges. Journal of Applied Crystallography, 46, 1407-1413. https://doi.org/10.1107/S0021889813022450

[28] Michels, R., Goerigk, G., Vainio, U., Gummel, J. and Huber, K. (2014) Coaggregation of Two Anionic Azo Dyestuffs: A Combined Static Light Scattering and Small-Angle X-Ray Scattering Study. The Journal of Physical Chemistry B, 118, 7618-7629. https://doi.org/10.1021/jp502347b

[29] Weber, C., Reichenauer, G., and Pflaum, J. (2015) Electroless Preparation and ASAXS Microstructural Analysis of Pseudocapacitive Carbon Manganese Oxide Supercapacitor Electrodes. Langmuir, 31, 782-788. https://doi.org/10.1021/la5027762

[30] Goerigk, G., Lages, S. and Huber, K. (2016) Systematic Limitations in Concentration Analysis via Anomalous Small-Angle X-Ray Scattering in the Small Structure 
Limit. Polymers, 8, 1-15. https://doi.org/10.3390/polym8030085

[31] Stuhrmann, H.B. (1985) Resonance Scattering in Macromolecular Structure Research. In: Kausch, H.H. and Zachmann, H.G., Eds., Characterization of Polymers in the Solid State II: Synchrotron Radiation, X-Ray Scattering and Electron Microscopy. Advances in Polymer Science, Vol. 67, Springer-Verlag, Berlin, 123-163. https://doi.org/10.1007/BFb0016608

[32] Higashi, M., Domen, K. and Abe, R. (2011) Fabrication of Efficient TaON and $\mathrm{Ta}_{3} \mathrm{~N}_{5}$ Photoanodes for Water Splitting under Visible Light Irradiation. Energy \& Environmental Science, 4, 4138. https://doi.org/10.1039/c1ee01878g

[33] Westlake, J.R. (1968) A Handbook of Numerical Matrix Inversion and Solution of Linear Equations. John Wiley \& Sons, New York.

[34] Buth, G. (1992) Untersuchung der Mikrostruktur magnetooptischer Speicherschichten mit Röntgenkleinwinkelstreuung unter Anwendung der Kontrastvariationsmethode. RWTH Aachen, Aachen.

[35] Cromer, D.T. and Liberman, D. (1970) Relativistic Calculation of Anomalous Scattering Factors for X Rays. The Journal of Chemical Physics, 53, 1891-1898.

https://doi.org/10.1063/1.1674266

[36] Cromer, D.T. and Liberman, D. (1981) Anomalous Dispersion Calculations Near to and on the Long-Wavelength Side of an Absorption Edge. Acta Crystallographica, A37, 267-268. https://doi.org/10.1107/S0567739481000600 


\section{Appendix 1: Details of the General Matrix Equation}

\section{Established by Anomalous Small-Angle X-Ray Scattering}

The three scattering contributions obtained via inserting Equation (3) into equation (2) and subsequent averaging over all orientations are:

$$
\begin{aligned}
& S_{A}(q, \varepsilon)=\left|\Delta f_{A}(\varepsilon)\right|^{2} \iint_{V_{p}} v_{A}(\overrightarrow{\mathbf{r}}) v_{A}\left(\overrightarrow{\mathbf{r}}^{\prime}\right) \frac{\sin \left(q\left|\overrightarrow{\mathbf{r}}-\overrightarrow{\mathbf{r}}^{\prime}\right|\right)}{q\left|\overrightarrow{\mathbf{r}}-\overrightarrow{\mathbf{r}}^{\prime}\right|} \mathrm{d}^{3} r \mathrm{~d}^{3} r^{\prime} \\
& S_{A B}(q, \varepsilon)=2\left[\left(f_{0, A}-\rho V_{A}+f_{A}^{\prime}(\varepsilon)\right) \cdot\left(f_{0, B}-\rho V_{B}+f_{B}^{\prime}(\varepsilon)\right)+f_{A}^{\prime \prime}(\varepsilon) f_{B}^{\prime \prime}(\varepsilon)\right] \\
& \cdot \iint_{V_{p}} v_{A}(\overrightarrow{\mathbf{r}}) v_{B}\left(\overrightarrow{\mathbf{r}}^{\prime}\right) \frac{\sin \left(q\left|\overrightarrow{\mathbf{r}}-\overrightarrow{\mathbf{r}}^{\prime}\right|\right)}{q\left|\overrightarrow{\mathbf{r}}-\overrightarrow{\mathbf{r}}^{\prime}\right|} \mathrm{d}^{3} r \mathrm{~d}^{3} r^{\prime} \\
& S_{B}(q, \varepsilon)=\left|\Delta f_{B}(\varepsilon)\right|^{2} \iint_{V_{p}} v_{B}(\overrightarrow{\mathbf{r}}) v_{B}\left(\overrightarrow{\mathbf{r}}^{\prime}\right) \frac{\sin \left(q\left|\overrightarrow{\mathbf{r}}-\overrightarrow{\mathbf{r}}^{\prime}\right|\right)}{q\left|\overrightarrow{\mathbf{r}}-\overrightarrow{\mathbf{r}}^{\prime}\right|} \mathrm{d}^{3} r \mathrm{~d}^{3} r^{\prime}
\end{aligned}
$$

The convolution integrals represent the Fourier transform of the pair correlation functions. When taking out the chemical concentrations from the convolution integrals we receive:

$$
\begin{aligned}
S_{A}(q, \varepsilon)= & \left|\Delta f_{A}(\varepsilon)\right|^{2} / V_{A}^{2} \varphi_{A}\left(1-\varphi_{A}\right) \iint_{V_{p}} \gamma_{A}(\overrightarrow{\mathbf{r}}) \gamma_{A}\left(\overrightarrow{\mathbf{r}}^{\prime}\right) \frac{\sin \left(q\left|\overrightarrow{\mathbf{r}}-\overrightarrow{\mathbf{r}}^{\prime}\right|\right)}{q\left|\overrightarrow{\mathbf{r}}-\overrightarrow{\mathbf{r}}^{\prime}\right|} \mathrm{d}^{3} r \mathrm{~d}^{3} r^{\prime} \\
= & \left|\Delta f_{A}(\varepsilon)\right|^{2} / V_{A}^{2} \varphi_{A}\left(1-\varphi_{A}\right)\left|A_{A}(q)\right|^{2} \\
S_{A B}(q, \varepsilon)= & 2\left[\left(f_{0, A}-\rho V_{A}+f_{A}^{\prime}(\varepsilon)\right) \cdot\left(f_{0, B}-\rho V_{B}+f_{B}^{\prime}(\varepsilon)\right)\right. \\
& \left.+f_{A}^{\prime \prime}(\varepsilon) f_{B}^{\prime \prime}(\varepsilon)\right] / V_{A} / V_{B} \varphi_{A} \varphi_{B} \cdot \iint_{V_{p}} \gamma_{A}(\overrightarrow{\mathbf{r}}) \gamma_{B}\left(\overrightarrow{\mathbf{r}}^{\prime}\right) \frac{\sin \left(q\left|\overrightarrow{\mathbf{r}}-\overrightarrow{\mathbf{r}}^{\prime}\right|\right)}{q\left|\overrightarrow{\mathbf{r}}-\overrightarrow{\mathbf{r}}^{\prime}\right|} \mathrm{d}^{3} r \mathrm{~d}^{3} r^{\prime} \\
= & 2\left[\left(f_{0, A}-\rho V_{A}+f_{A}^{\prime}(\varepsilon)\right) \cdot\left(f_{0, B}-\rho V_{B}+f_{B}^{\prime}(\varepsilon)\right)\right. \\
& \left.+f_{A}^{\prime \prime}(\varepsilon) f_{B}^{\prime \prime}(\varepsilon)\right] / V_{A} / V_{B} \varphi_{A} \varphi_{B} \cdot \operatorname{Re}\left(A_{A}(q) A_{B}(q)\right) \\
S_{B}(q, \varepsilon)= & \left|\Delta f_{B}(\varepsilon)\right|^{2} / V_{B}^{2} \varphi_{B}\left(1-\varphi_{B}\right) \iint_{V_{p}} \gamma_{B}(\overrightarrow{\mathbf{r}}) \gamma_{B}\left(\overrightarrow{\mathbf{r}}^{\prime}\right) \frac{\sin \left(q\left|\overrightarrow{\mathbf{r}}-\overrightarrow{\mathbf{r}}^{\prime}\right|\right)}{q\left|\overrightarrow{\mathbf{r}}-\overrightarrow{\mathbf{r}}^{\prime}\right|} \mathrm{d}^{3} r \mathrm{~d}^{3} r^{\prime} \\
=\mid & \left|\Delta f_{B}(\varepsilon)\right|^{2} / V_{B}^{2} \varphi_{B}\left(1-\varphi_{B}\right)\left|A_{B}(q)\right|^{2}
\end{aligned}
$$

The functions $\gamma_{A}(\overrightarrow{\mathbf{r}}), \gamma_{B}(\overrightarrow{\mathbf{r}})$ are the normalized density functions of the atomic (molecular) species $\mathrm{A}$ and $\mathrm{B}$, respectively and represent their spatial distribution in the sample. Thus the functions $\left|A_{A}(q)\right|^{2}, \operatorname{Re}\left(A_{A}(q) A_{B}(q)\right),\left|A_{B}(q)\right|^{2}$ represent the normalized pair correlation functions.

The matrix coefficients and vector components obtained from Equation (7) via elementary transformations are:

$$
\begin{aligned}
& u_{11}\left(\varepsilon_{1}\right)=a_{11}\left(\varepsilon_{1}\right) \\
& u_{12}\left(\varepsilon_{1}\right)=a_{12}\left(\varepsilon_{1}\right) \\
& u_{13}\left(\varepsilon_{1}\right)=a_{13}\left(\varepsilon_{1}\right) \\
& u_{22}\left(\varepsilon_{1}, \varepsilon_{2}\right)=a_{22}\left(\varepsilon_{2}\right)-a_{21}\left(\varepsilon_{2}\right) / a_{11}\left(\varepsilon_{1}\right) \cdot a_{12}\left(\varepsilon_{1}\right) \\
& u_{23}\left(\varepsilon_{1}, \varepsilon_{2}\right)=a_{23}\left(\varepsilon_{2}\right)-a_{21}\left(\varepsilon_{2}\right) / a_{11}\left(\varepsilon_{1}\right) \cdot a_{13}\left(\varepsilon_{1}\right)
\end{aligned}
$$




$$
\begin{aligned}
& u_{33}\left(\varepsilon_{1}, \varepsilon_{2}, \varepsilon_{3}\right)=\left(a_{33}\left(\varepsilon_{3}\right)-a_{31}\left(\varepsilon_{3}\right) / a_{11}\left(\varepsilon_{1}\right) \cdot a_{13}\left(\varepsilon_{1}\right)\right) \\
& -\left(a_{32}\left(\varepsilon_{3}\right)-a_{31}\left(\varepsilon_{3}\right) / a_{11}\left(\varepsilon_{1}\right) \cdot a_{12}\left(\varepsilon_{1}\right)\right) /\left(a_{22}\left(\varepsilon_{2}\right)-a_{21}\left(\varepsilon_{2}\right) / a_{11}\left(\varepsilon_{1}\right) \cdot a_{12}\left(\varepsilon_{1}\right)\right) \\
& \cdot\left(a_{23}\left(\varepsilon_{2}\right)-a_{21}\left(\varepsilon_{2}\right) / a_{11}\left(\varepsilon_{1}\right) \cdot a_{13}\left(\varepsilon_{1}\right)\right) \\
& B_{1}\left(q, \varepsilon_{1}\right)=I\left(q, \varepsilon_{1}\right) \\
& B_{2}\left(q, \varepsilon_{1,2}\right)=I\left(q, \varepsilon_{2}\right)-a_{21}\left(\varepsilon_{2}\right) / a_{11}\left(\varepsilon_{1}\right) \cdot I\left(q, \varepsilon_{1}\right) \\
& B_{3}\left(q, \varepsilon_{1,2,3}\right)=\left(I\left(q, \varepsilon_{3}\right)-a_{31}\left(\varepsilon_{3}\right) / a_{11}\left(\varepsilon_{1}\right) \cdot I\left(q, \varepsilon_{1}\right)\right) \\
& -\left(a_{32}\left(\varepsilon_{3}\right)-a_{31}\left(\varepsilon_{3}\right) / a_{11}\left(\varepsilon_{1}\right) \cdot a_{12}\left(\varepsilon_{1}\right)\right) /\left(a_{22}\left(\varepsilon_{2}\right)-a_{21}\left(\varepsilon_{2}\right) / a_{11}\left(\varepsilon_{1}\right) \cdot a_{12}\left(\varepsilon_{1}\right)\right) \\
& \cdot\left(I\left(q, \varepsilon_{2}\right)-a_{21}\left(\varepsilon_{2}\right) / a_{11}\left(\varepsilon_{1}\right) \cdot I\left(q, \varepsilon_{1}\right)\right)
\end{aligned}
$$

Generally two possibilities exist to calculate the condition numbers of a SLE constituted by ASAXS. Starting with the original matrix

$$
\left(\begin{array}{lll}
a_{11} & a_{12} & a_{13} \\
a_{21} & a_{22} & a_{23} \\
a_{31} & a_{32} & a_{33}
\end{array}\right) \otimes\left(\begin{array}{c}
\left|A_{A}(q)\right|^{2} \\
\operatorname{Re}\left(A_{A}(q) A_{B}(q)\right) \\
\left|A_{B}(q)\right|^{2}
\end{array}\right)=\left(\begin{array}{c}
I\left(q, \varepsilon_{1}\right) \\
I\left(q, \varepsilon_{2}\right) \\
I\left(q, \varepsilon_{3}\right)
\end{array}\right)
$$

or using the upper triangular matrix:

$$
\left(\begin{array}{ccc}
u_{11} & u_{12} & u_{13} \\
0 & u_{22} & u_{23} \\
0 & 0 & u_{33}
\end{array}\right) \otimes\left(\begin{array}{c}
\left|A_{A}(q)\right|^{2} \\
\operatorname{Re}\left(A_{A}(q) A_{B}(q)\right) \\
\left|A_{B}(q)\right|^{2}
\end{array}\right)=\left(\begin{array}{c}
B_{1} \\
B_{2} \\
B_{3}
\end{array}\right)
$$

When using (A5) better (smaller) $N$-conditions are calculated but at the same time the errors of the right hand vector increase due to error propagation because:

$$
\mathbf{L}^{-1} \otimes \mathbf{I}=\mathbf{B}
$$

In summary better results are obtained from starting with (A5). A detailed analysis of this problem can be found in [25]. 


\section{Appendix 2: Details of the Mathematical Algorithm Employed in ASAXS Techniques (Jacobian)-Error Propagation}

Equations (11)-(12) combine scattering curves from three different X-ray energies (wavelengths). Because the scattering vector $q$ depends on the wavelength corresponding $q$-values are located at different detector coordinates. This makes the introduction of the Jacobian necessary for the transformation from Cartesian (detector related pixel) coordinates into polar coordinates in the reciprocal space in order to receive scattering curves with identical $q$-binning:

$$
\begin{aligned}
I(q(x, y, z)) & =\int_{q_{k}(x, y, z)}^{q_{k}+\Delta q} \frac{\mathrm{d} \sigma}{\mathrm{d} \Omega}(q(x, y, z)) \mathrm{d}^{3} q \\
& =\int_{q_{k}(x, y, z)}^{q_{k}+\Delta q} \frac{\mathrm{d} \sigma}{\mathrm{d} \Omega}(q(x, y, z)) \frac{\partial\left(q_{r}, q_{\vartheta}, q_{\varphi}\right)}{\partial(x, y, z)} d^{3} r \\
& =\int_{\vartheta} \int_{\varphi}^{q_{k}+\Delta q} \int_{q_{k}(x, y, z)}^{\mathrm{d} \sigma} \frac{\mathrm{d} \Omega}{(q(x, y, z)) \sin \vartheta \mathrm{d} \vartheta \mathrm{d} \phi q^{2} \mathrm{~d} q} \\
& =2 \int_{q_{k}+\Delta q}^{q_{k}(x, y, z)} \frac{\mathrm{d} \sigma}{\mathrm{d} \Omega}(q(x, y, z)) \mathrm{d} q^{2} \\
\mathrm{~d} q^{2}=q \mathrm{~d} q \mathrm{~d} \phi & =\left(\frac{2 \pi}{\lambda l}\right)^{2} \frac{2+r^{2}+2 \sqrt{1+r^{2}}}{\left(1+r^{2}+\sqrt{1+r^{2}}\right)^{2} \sqrt{1+r^{2}}} \mathrm{~d} x \mathrm{~d} y \\
r=\frac{\sqrt{x^{2}+y^{2}}}{l} &
\end{aligned}
$$

where $\mathrm{d} \sigma / \mathrm{d} \Omega$ represents the scattering cross sections with error bars stored in the detector pixel with the cartesian coordinates $x, y, z$, and $l$ is the sample to detector distance. $\partial\left(q_{r}, q_{\vartheta}, q_{\varphi}\right) / \partial(x, y, z)$ is the Jacobian. The integration limits of the $k$-th $q$-interval are functions of the cartesian coordinates $\vartheta(x, y, z), \varphi(x, y, z), q_{k}(x, y, z)$. The error bars of $\mathrm{d} \sigma / \mathrm{d} \Omega$ have been calculated via error propagation from the photon counts in the detector pixel of the single photon count detector. Via (A7) the error propagation from the detector pixel to the $q$-intervals can be calculated. The mathematical details are outlined in [34]. The Jacobian represents the exact coordinate transformation establishing the same $q$-binning for scattering curves measured at different wavelengths, which is inevitably required when applying Equation (12). Other non-linear algorithms (for instance interpolation via splines) introduce large errors thereby disabling the matrix inversion and the application of Equation (12) with a subsequent calculation of error propagation. 


\section{Appendix 3: Details of the Calculations of the Condition Numbers}

The two-dimensional maps of $N(\mathrm{U})$ of Figures 1-3 (depicted in the plane spanned by the electron density respective electron contrast and the atomic number) have been calculated via Equation (13) using C-code and subsequently are displayed

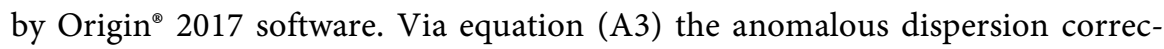
tions [35] [36] of Equation (7) enter the $N$-condition. The $N$-condition shows strong differences depending on the input parameters listed in the Tables A1-A3 for three examples. The chemical elements have different dispersion corrections but the values are similar for $\mathrm{K}$ - respective $\mathrm{L}_{\mathrm{III}}$-absorption edges. Thus in order to generalize the calculated maps of Figures 1-3 dispersion parameters preferentially used in ASAXS experiments at $\mathrm{K}$ - respective $\mathrm{L}_{\mathrm{III}}$-absorption edges have been assumed.

As can be seen from Table A3 the anomalous dispersion corrections of the $\mathrm{L}_{\mathrm{III}}$-absorption edges provide higher differences in combination with higher $f^{\text {, }}$ values. In consequence this provides higher significance of ASAXS experiments performed at $\mathrm{L}_{\mathrm{III}}$-edges when compared to K-edges.

Table A1. Anomalous dispersion corrections typical for ASAXS experiments of two-phase systems performed in the energy range of K-absorption edges not using the energy of the absorption edge. Ge serves as an example [17].

\begin{tabular}{ccc}
\hline \multirow{2}{*}{ Energy [eV] } & \multicolumn{3}{c}{ K-absorption edge of Ge } \\
\cline { 2 - 3 } & $f^{\prime}$ & $f^{\prime}$ \\
\hline$\varepsilon_{1}: 10053$ & -2.093 & 0.592 \\
$\varepsilon_{2}: 11083$ & -5.996 & 0.495 \\
$\varepsilon_{3}: 11098$ & -7.458 & 0.493 \\
\hline
\end{tabular}

Table A2. Anomalous dispersion corrections typical for ASAXS experiments of three-phase systems performed in the energy range of K-absorption edges using the energy of the absorption edge. Sr serves as an example [20].

\begin{tabular}{ccc}
\hline \multirow{2}{*}{ Energy $[\mathrm{eV}]$} & \multicolumn{2}{c}{ K-absorption edge of Sr } \\
\cline { 2 - 3 } & $f$ & $f^{\prime}$ \\
\hline$\varepsilon_{1}: 15507$ & -2.75 & 0.56 \\
$\mathcal{\varepsilon}_{2}: 16093$ & -6.66 & 0.53 \\
$\varepsilon_{3}: 16105$ & -10.3 & 2.08 \\
\hline
\end{tabular}

Table A3. Anomalous dispersion corrections typical for ASAXS experiments of three-phase systems performed in the energy range of $\mathrm{L}_{\mathrm{III}}$-absorption edges using the energy of the absorption edge. $\mathrm{Pb}$ serves as an example [30].

\begin{tabular}{ccc}
\hline \multirow{2}{*}{ Energy $[\mathrm{eV}]$} & \multicolumn{2}{c}{$\mathrm{L}_{\mathrm{III}}$-absorption edge of $\mathrm{Pb}$} \\
\cline { 2 - 3 } & $\mathrm{f}$ & $\mathrm{f}^{\prime}$ \\
\hline$\varepsilon_{1}: 12650$ & -9.982 & 4.134 \\
$\varepsilon_{2}: 13000$ & -14.602 & 3.956 \\
$\varepsilon_{3}: 13035$ & -22.666 & 6.002 \\
\hline
\end{tabular}

